\title{
Testing of MTF replacement dynamic model for model example
}

\author{
Arthur Alukhanyan ${ }^{1, *}$, Olga Panfilova ${ }^{1}$, and Vadim Alukhanyan ${ }^{1}$ \\ ${ }^{1}$ Don State Technical University, 1, sq. Gagarina, 3440003, Rostov-on-Don, Russia
}

\begin{abstract}
The article is devoted to testing of economic and mathematical model for reequipment of machine-tractor fleet (MTF) of agricultural enterprise using the model example simulating real technological and economical processes used at the enterprise. Moreover, the daily capacity of machines, schedule of optimum agrotechnical terms for operations, etc. are given as basic examples of the technological process in the model example. Moreover, the algorithm for correction of optimum solving consisting of eight steps is given in the research. The value of the next controlled variable by its rounding to the integer value is recorded at each step of the algorithm.
\end{abstract}

\section{Introduction}

The issues of mathematical modelling of economic processes are quite well covered in the domestic and foreign scientific literature. In particular, we are talking about the problems of technical re-equipment of the machine and tractor fleet (MTF) of agricultural enterprises, which are successfully solved using optimization models. In some scientific articles (1), economic and mathematical models are presented that allow minimizing the agro technical deadlines for performing agricultural operations. In other works $(2,3)$, an MTF management system is being developed to improve the efficiency of agricultural enterprises. They note that the cost of buying new equipment is one of the main and quite significant, which is due to the high level of mechanization of agricultural production. Among the possible sources of financing for the technical re-equipment of the MTF, the authors distinguish: own funds, leasing (4), credit, etc. In some works (5-7), the task of increasing productivity (daily and seasonal) is solved) MTF, which has a positive impact on the efficiency of agricultural production.

However, most studies do not address the issue of testing economic and mathematical models of MTF management on model examples, which is necessary to improve the objectivity and stability of these models in real business conditions.

\section{Materials and Methods}

In accordance with (8), for the problem of technical re-equipment of the MTF (9-16), we have a linear programming problem. Let's the process complex consists of three operations $(i=1,2,3)$, in the fleet there are two types of machines $(j=1,2)$. The standard operation

\footnotetext{
*Corresponding author: aaalukhanyan78@mail.ru
} 
time is 5 years for the machine of the first type, 7 years for the machine of the second type $\left(\bar{t}_{1}=5, \bar{t}_{2}=7\right)$. Age composition of the equipment in the fleet and machine capacity are given in Tables 1 and 2 accordingly.

Table 1. Age Composition (Quantity) of Equipment, pcs.

\begin{tabular}{|c|c|c|c|c|c|}
\hline \multirow{2}{*}{ Machine Type } & \multicolumn{5}{|c|}{ Age, years } \\
\cline { 2 - 6 } & $\mathbf{3}$ & $\mathbf{4}$ & $\mathbf{5}$ & $\mathbf{6}$ & $\mathbf{7}$ \\
\hline $\mathbf{1}$ & 2 & 4 & 5 & 0 & 0 \\
\hline $\mathbf{2}$ & 0 & 1 & 3 & 1 & 2 \\
\hline
\end{tabular}

Table 2. Daily Capacity of Machines, ha/day.

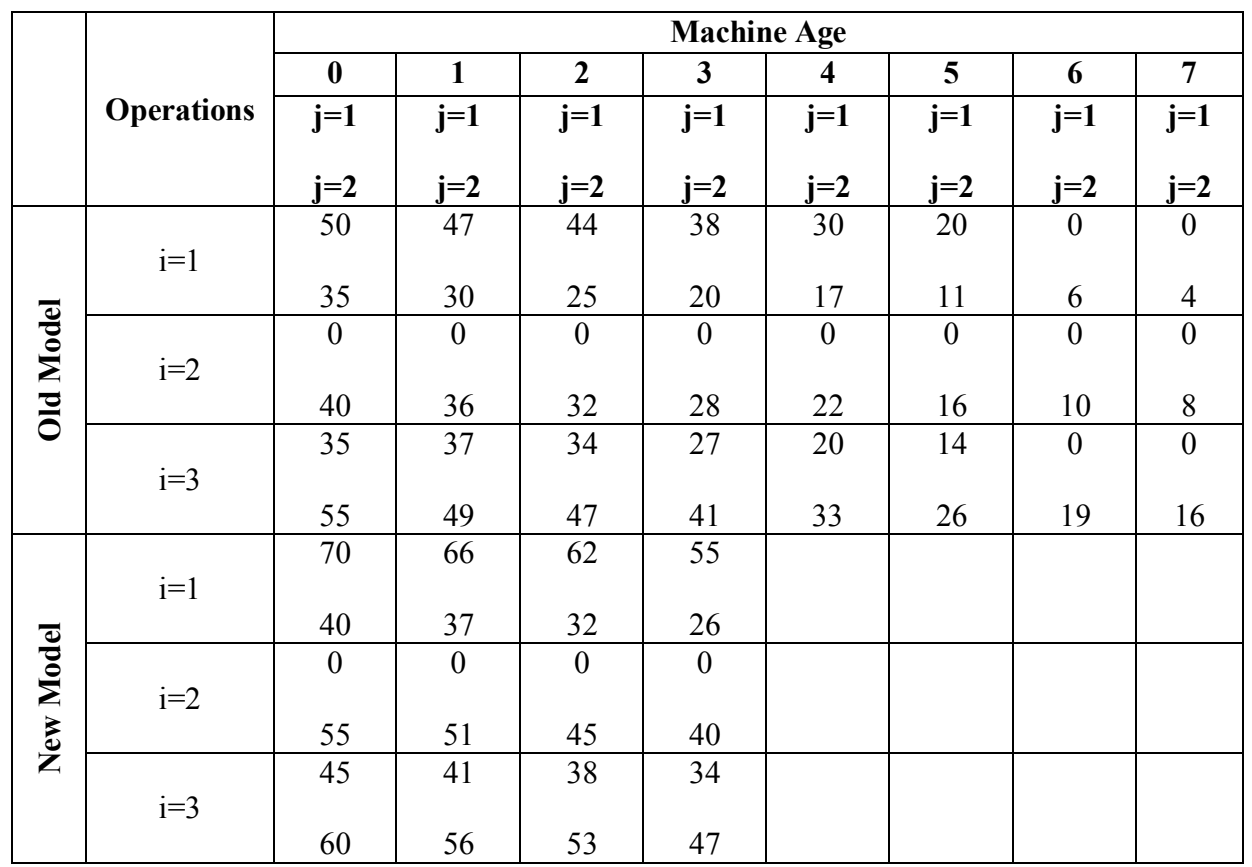

Table 3 shows the information concerning the residual value of the machine of old modification.

Table 3. Residual Value of Machines of Old Modification, monetary unit.

\begin{tabular}{|c|c|c|c|c|c|c|c|c|}
\hline \multirow{2}{*}{$\begin{array}{c}\text { Machine } \\
\text { Type }\end{array}$} & \multicolumn{9}{|c|}{ Machine Age } \\
\cline { 2 - 9 } & $\mathbf{0}$ & $\mathbf{1}$ & $\mathbf{2}$ & $\mathbf{3}$ & $\mathbf{4}$ & $\mathbf{5}$ & $\mathbf{6}$ & $\mathbf{7}$ \\
\hline $\mathrm{j}=1$ & 100 & 80 & 60 & 40 & 20 & 0 & & \\
\hline $\mathrm{j}=2$ & 140 & 120 & 100 & 80 & 60 & 40 & 20 & 0 \\
\hline
\end{tabular}

In the example the planning horizon is equal to two years (seasons). Prices for new models of the machines of both types are given below. Year of acquisition is indicated in the brackets. In the second year of the account period 5\% increase of prices for machines is supposed. Thus:

$$
\begin{aligned}
& \tilde{c}_{1}(1)=150 ; \tilde{c}_{1}(2)=157 ; \\
& \tilde{c}_{2}(1)=180 ; \tilde{c}_{2}(2)=189 .
\end{aligned}
$$

As previously stated data on the technological process includes the required capacity and optimum agrotechnical terms for work performance by operations. Let's capacity by 
operations is 500,700 and 1000 ha accordingly for the first, second and third operations. It is assumed that the structure of cropped lands remain unchanged within the second year.

The optimum agrotechnical terms for performance of the whole process complex (all three operations) are equal to seven days since August 1st till 7th inclusively. Moreover for the first operation the period since August 1st till 3rd is optimum, for the second operation the period since August 3rd till 5th is optimum, and for the third operation August 6th, 7th is optimum. Thus, the table of optimum agrotechnical terms is as follows.

Table 4. Schedule of Optimum agrotechnical terms by Operations.

\begin{tabular}{|c|c|c|c|c|c|c|c|}
\hline & 01.08 & 02.08 & 03.08 & 04.08 & 05.08 & 06.08 & 07.08 \\
\hline $\mathrm{i}=1$ & & & & & & & \\
\hline $\mathrm{i}=2$ & & & & & & & \\
\hline $\mathrm{i}=3$ & & & & & & & \\
\hline
\end{tabular}

Data concerning the average annual operating costs are given in Table 5. For simplicity it is assumed that the operating costs depend only on the machine type, its modification and age. Therefore, the possible increase of prices for fuel, specialty fluids, etc. in the course of time is not considered.

Table 5. Average Annual Operating Costs for Machines, monetary unit.

\begin{tabular}{|c|c|c|c|c|c|c|c|c|c|}
\hline & \multirow{2}{*}{$\begin{array}{c}\text { Machine } \\
\text { Types }\end{array}$} & \multicolumn{8}{|c|}{ Machine Age (Number of Years in Operation) } \\
\hline & & $\mathbf{0}$ & 1 & 2 & 3 & 4 & 5 & 6 & 7 \\
\hline \multirow{2}{*}{$\begin{array}{l}\overline{0} \\
\frac{\bar{\delta}}{0} \\
\frac{0}{0}\end{array}$} & $\mathrm{j}=1$ & 7 & 8 & 10 & 13 & 16 & 19 & & \\
\hline & $\mathrm{j}=2$ & 10 & 12 & 14 & 18 & 23 & 28 & 35 & 40 \\
\hline \multirow{2}{*}{$\begin{array}{l}\overline{0} \\
\sum_{0}^{0} \\
z \\
z \\
z\end{array}$} & $\mathrm{j}=1$ & 12 & 15 & & & & & & \\
\hline & $j=2$ & 16 & 20 & & & & & & \\
\hline
\end{tabular}

At simple task setting the possibility of financing for fleet reequipment is restricted only with self-financing (due to free funds and from depreciation reserve). Let's maximum 600 and 900 monetary units accordingly within the first and second years of the account period could be spent for implementation of the appropriate measures on fleet renewal. In calculations we will use the discount rate to be $20 \%$. The description of the controlled variables is given in Table 6 .

Table 6. Controllable Variables of the Problem.

\begin{tabular}{|c|c|c|c|c|c|c|c|}
\hline No. & $\begin{array}{c}\tilde{\boldsymbol{x}}_{\boldsymbol{j}}(\boldsymbol{m}) . \\
\tilde{\boldsymbol{x}}_{\boldsymbol{i} \boldsymbol{j}}^{\boldsymbol{k}}(\boldsymbol{m})\end{array}$ & No. & $\boldsymbol{n}_{\boldsymbol{i j}}^{\boldsymbol{k}}(\boldsymbol{t} . \boldsymbol{m})$ & No. & $\boldsymbol{n}_{\boldsymbol{i j}}^{\boldsymbol{k}}(\boldsymbol{t} . \boldsymbol{m})$ & No. & $\begin{array}{c}\boldsymbol{n}_{\boldsymbol{i} \boldsymbol{j}}^{\boldsymbol{k}}(\boldsymbol{t} . \boldsymbol{m}) \mathbf{.} \\
\boldsymbol{y}_{\boldsymbol{j}}(\boldsymbol{t} . \boldsymbol{m})\end{array}$ \\
\hline 1. & $\tilde{x}_{1}(1)$ & 31. & $n_{11}^{1}(3.1)$ & 61. & $n_{32}^{7}(6.1)$ & 91. & $n_{22}^{3}(7.2)$ \\
\hline 2. & $\tilde{x}_{2}(1)$ & 32. & $n_{11}^{2}(3.1)$ & 62. & $n_{12}^{1}(7.1)$ & 92. & $n_{22}^{4}(7.2)$ \\
\hline 3. & $\tilde{x}_{1}(2)$ & 33. & $n_{11}^{3}(3.1)$ & 63. & $n_{12}^{2}(7.1)$ & 93. & $n_{22}^{5}(7.2)$ \\
\hline 4. & $\tilde{x}_{2}(2)$ & 34. & $n_{31}^{6}(3.1)$ & 64. & $n_{12}^{3}(7.1)$ & 94. & $n_{32}^{6}(7.2)$ \\
\hline 5. & $\tilde{x}_{11}^{1}(1)$ & 35. & $n_{31}^{7}(3.1)$ & 65. & $n_{22}^{3}(7.1)$ & 95. & $n_{32}^{7}(7.2)$ \\
\hline 6. & $\tilde{x}_{11}^{2}(1)$ & 36. & $n_{11}^{1}(4.1)$ & 66. & $n_{22}^{4}(7.1)$ & 96. & $y_{1}(3.1)$ \\
\hline 7. & $\tilde{x}_{11}^{3}(1)$ & 37. & $n_{11}^{2}(4.1)$ & 67. & $n_{22}^{5}(7.1)$ & 97. & $y_{1}(4.1)$ \\
\hline 8. & $\tilde{x}_{31}^{6}(1)$ & 38. & $n_{11}^{3}(4.1)$ & 68. & $n_{32}^{6}(7.1)$ & 98. & $y_{1}(5.1)$ \\
\hline
\end{tabular}




\begin{tabular}{|c|c|c|c|c|c|c|c|}
\hline 9. & $\tilde{x}_{31}^{7}(1)$ & 39. & $n_{31}^{6}(4.1)$ & 69. & $n_{32}^{7}(7.1)$ & 99. & $y_{2}(5.1)$ \\
\hline 10. & $\tilde{x}_{12}^{1}(1)$ & 40. & $n_{31}^{7}(4.1)$ & 70. & $n_{11}^{1}(4.2)$ & 100. & $y_{2}(6.1)$ \\
\hline 11. & $\tilde{x}_{12}^{2}(1)$ & 41. & $n_{11}^{1}(5.1)$ & 71. & $n_{11}^{2}(4.2)$ & 101. & $y_{2}(7.1)$ \\
\hline 12. & $\tilde{x}_{12}^{3}(1)$ & 42. & $n_{11}^{2}(5.1)$ & 72. & $n_{11}^{3}(4.2)$ & 102. & $y_{1}(4.2)$ \\
\hline 13. & $\tilde{x}_{22}^{3}(1)$ & 43. & $n_{11}^{3}(5.1)$ & 73. & $n_{31}^{6}(4.2)$ & 103. & $y_{1}(5.2)$ \\
\hline 14. & $\tilde{x}_{22}^{4}(1)$ & 44. & $n_{31}^{6}(5.1)$ & 74. & $n_{31}^{7}(4.2)$ & 104. & $y_{2}(6.2)$ \\
\hline 15. & $\tilde{x}_{22}^{5}(1)$ & 45. & $n_{31}^{7}(5.1)$ & 75. & $n_{11}^{1}(5.2)$ & 105. & $y_{2}(7.2)$ \\
\hline 16. & $\tilde{x}_{32}^{6}(1)$ & 46. & $n_{12}^{1}(5.1)$ & 76. & $n_{11}^{2}(5.2)$ & & \\
\hline 17. & $\tilde{x}_{32}^{7}(1)$ & 47. & $n_{12}^{2}(5.1)$ & 77. & $n_{11}^{3}(5.2)$ & & \\
\hline 18. & $\tilde{x}_{11}^{1}(2)$ & 48. & $n_{12}^{3}(5.1)$ & 78. & $n_{31}^{6}(5.2)$ & & \\
\hline 19. & $\tilde{x}_{11}^{2}(2)$ & 49. & $n_{22}^{3}(5.1)$ & 79. & $n_{31}^{7}(5.2)$ & & \\
\hline 20. & $\tilde{x}_{11}^{3}(2)$ & 50. & $n_{22}^{4}(5.1)$ & 80. & $n_{12}^{1}(6.2)$ & & \\
\hline 21. & $\tilde{x}_{31}^{6}(2)$ & 51. & $n_{22}^{5}(5.1)$ & 81. & $n_{12}^{2}(6.2)$ & & \\
\hline 22. & $\tilde{x}_{31}^{7}(2)$ & 52. & $n_{32}^{6}(5.1)$ & 82. & $n_{12}^{3}(6.2)$ & & \\
\hline 23. & $\tilde{x}_{12}^{1}(2)$ & 53. & $n_{32}^{7}(5.1)$ & 83. & $n_{22}^{3}(6.2)$ & & \\
\hline 24. & $\tilde{x}_{12}^{2}(2)$ & 54. & $n_{12}^{1}(6.1)$ & 84. & $n_{22}^{4}(6.2)$ & & \\
\hline 25. & $\tilde{x}_{12}^{3}(2)$ & 55. & $n_{12}^{2}(6.1)$ & 85. & $n_{22}^{5}(6.2)$ & & \\
\hline 26. & $\tilde{x}_{22}^{3}(2)$ & 56. & $n_{12}^{3}(6.1)$ & 86. & $n_{32}^{6}(6.2)$ & & \\
\hline 27. & $\tilde{x}_{22}^{4}(2)$ & 57. & $n_{22}^{3}(6.1)$ & 87. & $n_{32}^{7}(6.2)$ & & \\
\hline 28. & $\tilde{x}_{22}^{5}(2)$ & 58. & $n_{22}^{4}(6.1)$ & 88. & $n_{12}^{1}(7.2)$ & & \\
\hline 29. & $\tilde{x}_{32}^{6}(2)$ & 59. & $n_{22}^{5}(6.1)$ & 89. & $n_{12}^{2}(7.2)$ & & \\
\hline 30. & $\tilde{x}_{32}^{7}(2)$ & 60. & $n_{32}^{6}(6.1)$ & 90. & $n_{12}^{3}(7.2)$ & & \\
\hline & & & & & & \\
\hline
\end{tabular}

\section{Record of restrictions for the controlled variables}

Restrictions 2 (8):

$$
\begin{gathered}
\tilde{x}_{11}^{1}(1) \leq \tilde{x}_{1}(1) ; \quad \tilde{x}_{12}^{1}(1) \leq \tilde{x}_{2}(1) ; \\
\tilde{x}_{11}^{1}(2) \leq \tilde{x}_{1}(2) ; \quad \tilde{x}_{12}^{1}(2) \leq \tilde{x}_{2}(2) ; \\
\tilde{x}_{11}^{2}(1) \leq \tilde{x}_{1}(1) ; \quad \tilde{x}_{12}^{2}(1) \leq \tilde{x}_{2}(1) ; \\
\tilde{x}_{11}^{2}(2) \leq \tilde{x}_{1}(2) ; \quad \tilde{x}_{12}^{2}(2) \leq \tilde{x}_{2}(2) ; \\
\tilde{x}_{11}^{3}(1) \leq \tilde{x}_{1}(1) ; \quad \tilde{x}_{12}^{3}(1)+\tilde{x}_{22}^{3}(1) \leq \tilde{x}_{2}(1) ; \\
\tilde{x}_{11}^{3}(2) \leq \tilde{x}_{1}(2) ; \quad \tilde{x}_{12}^{3}(2)+\tilde{x}_{22}^{3}(2) \leq \tilde{x}_{2}(2) ; \\
\tilde{x}_{31}^{6}(1) \leq \tilde{x}_{1}(1) ; \quad \tilde{x}_{22}^{4}(1) \leq \tilde{x}_{2}(1) ; \\
\tilde{x}_{31}^{6}(2) \leq \tilde{x}_{1}(2) ; \quad \tilde{x}_{22}^{4}(2) \leq \tilde{x}_{2}(2) ; \\
\tilde{x}_{31}^{7}(1) \leq \tilde{x}_{1}(1) ; \quad \tilde{x}_{22}^{5}(1) \leq \tilde{x}_{2}(1) ; \\
\tilde{x}_{31}^{7}(2) \leq \tilde{x}_{1}(2) ; \quad \tilde{x}_{22}^{5}(2) \leq \tilde{x}_{2}(2) ; \\
\tilde{x}_{32}^{6}(1) \leq \tilde{x}_{2}(1) ; \\
\tilde{x}_{32}^{6}(2) \leq \tilde{x}_{2}(2) ; \\
\tilde{x}_{32}^{7}(1) \leq \tilde{x}_{32}^{7}(1) ;
\end{gathered}
$$




$$
\tilde{x}_{32}^{7}(2) \leq \tilde{x}_{2}(2) \text {. }
$$

For recording restrictions 3 (8), use 4 (8). Then restrictions 3 (8) will have the following final form:

$$
\sum_{i} n_{i j}^{k}(t, m) \leq N_{j}(t-m+1)-\sum_{t=t-m+1, m=1}^{t, m} y_{j}(t, m) .
$$

Restrictions 1 for our problem are given below.

$$
\begin{aligned}
& n_{11}^{1}(3,1) \leq 2-y_{1}(3,1) ; \quad n_{12}^{1}(5,1) \leq 3-y_{2}(5,1) ; \\
& n_{11}^{1}(4,1) \leq 4-y_{1}(4,1) ; \quad n_{12}^{1}(6,1) \leq 1-y_{2}(6,1) \text {; } \\
& n_{11}^{1}(5,1) \leq 5-y_{1}(5,1) ; \quad n_{12}^{1}(7,1) \leq 2-y_{2}(7,1) \text {; } \\
& n_{11}^{2}(3,1) \leq 2-y_{1}(3,1) ; \quad n_{12}^{2}(5,1) \leq 3-y_{2}(5,1) \text {; } \\
& n_{11}^{2}(4,1) \leq 4-y_{1}(4,1) ; \quad n_{12}^{2}(6,1) \leq 1-y_{2}(6,1) ; \\
& n_{11}^{2}(5,1) \leq 5-y_{1}(5,1) ; \quad n_{12}^{2}(7,1) \leq 2-y_{2}(7,1) ; \\
& n_{11}^{3}(3,1) \leq 2-y_{1}(3,1) ; \quad n_{12}^{3}(5,1)+n_{22}^{3}(5,1) \leq 3-y_{2}(5,1) \text {; } \\
& n_{11}^{3}(4,1) \leq 4-y_{1}(4,1) ; \quad n_{12}^{3}(6,1)+n_{22}^{3}(6,1) \leq 1-y_{2}(6,1) \text {; } \\
& n_{11}^{3}(5,1) \leq 5-y_{1}(5,1) ; \quad n_{12}^{3}(7,1)+n_{22}^{3}(7,1) \leq 2-y_{2}(7,1) \text {; } \\
& n_{22}^{4}(5,1) \leq 3-y_{2}(5,1) \text {; } \\
& n_{22}^{4}(6,1) \leq 1-y_{2}(6,1) \text {; } \\
& n_{22}^{4}(7,1) \leq 2-y_{2}(7,1) \text {; } \\
& n_{22}^{5}(5,1) \leq 3-y_{2}(5,1) \text {; } \\
& n_{22}^{5}(6,1) \leq 1-y_{2}(6,1) \text {; } \\
& n_{22}^{5}(7,1) \leq 2-y_{2}(7,1) \text {; } \\
& n_{31}^{6}(3,1) \leq 2-y_{1}(3,1) ; \quad n_{32}^{6}(5,1) \leq 3-y_{2}(5,1) \text {; } \\
& n_{31}^{6}(4,1) \leq 4-y_{1}(4,1) ; \quad n_{32}^{6}(6,1) \leq 1-y_{2}(6,1) \text {; } \\
& n_{31}^{6}(5,1) \leq 5-y_{1}(5,1) ; \quad n_{32}^{6}(7,1) \leq 2-y_{2}(7,1) \text {; } \\
& n_{31}^{7}(3,1) \leq 2-y_{1}(3,1) ; \quad n_{32}^{7}(5,1) \leq 3-y_{2}(5,1) \text {; } \\
& n_{31}^{7}(4,1) \leq 4-y_{1}(4,1) ; \quad n_{32}^{7}(6,1) \leq 1-y_{2}(6,1) \text {; } \\
& n_{31}^{7}(5,1) \leq 5-y_{1}(5,1) ; \quad n_{32}^{7}(7,1) \leq 2-y_{2}(7,1) \text {; } \\
& n_{11}^{1}(4,2) \leq 2-y_{1}(3,1)-y_{1}(4,2) ; n_{12}^{1}(6,2) \leq 3-y_{2}(5,1)-y_{2}(6,2) \text {; } \\
& n_{11}^{1}(5,2) \leq 4-y_{1}(4,1)-y_{1}(5,2) ; n_{12}^{1}(7,2) \leq 1-y_{2}(6,1)-y_{2}(7,2) \text {; } \\
& n_{11}^{2}(4,2) \leq 2-y_{1}(3,1)-y_{1}(4,2) ; n_{12}^{2}(6,2) \leq 3-y_{2}(5,1)-y_{2}(6,2) \text {; } \\
& n_{11}^{2}(5,2) \leq 4-y_{1}(4,1)-y_{1}(5,2) ; n_{12}^{2}(7,2) \leq 1-y_{2}(6,1)-y_{2}(7,2) \text {; } \\
& n_{11}^{3}(4,2) \leq 2-y_{1}(3,1)-y_{1}(4,2) ; \quad n_{12}^{3}(6,2)+n_{22}^{3}(6,2) \leq 3-y_{2}(5,1)-y_{2}(6,2) \text {; } \\
& n_{11}^{3}(5,2) \leq 4-y_{1}(4,1)-y_{1}(5,2) ; \quad n_{12}^{3}(7,2)+n_{22}^{3}(7,2) \leq 1-y_{2}(6,1)-y_{2}(7,2) \text {; } \\
& n_{31}^{6}(4,2) \leq 2-y_{1}(3,1)-y_{1}(4,2) ; n_{22}^{4}(6,2) \leq 3-y_{2}(5,1)-y_{2}(6,2) \text {; }
\end{aligned}
$$




$$
\begin{gathered}
n_{31}^{6}(5,2) \leq 4-y_{1}(4,1)-y_{1}(5,2) ; n_{22}^{4}(7,2) \leq 1-y_{2}(6,1)-y_{2}(7,2) \\
n_{31}^{7}(4,2) \leq 2-y_{1}(3,1)-y_{1}(4,2) ; n_{22}^{5}(6,2) \leq 3-y_{2}(5,1)-y_{2}(6,2) ; \\
n_{31}^{7}(5,2) \leq 4-y_{1}(4,1)-y_{1}(5,2) ; n_{22}^{5}(7,2) \leq 1-y_{2}(6,1)-y_{2}(7,2) ; \\
n_{32}^{6}(6,2) \leq 3-y_{2}(5,1)-y_{2}(6,2) ; \\
n_{32}^{6}(7,2) \leq 1-y_{2}(6,1)-y_{2}(7,2) ; \\
n_{32}^{7}(6,2) \leq 3-y_{2}(5,1)-y_{2}(6,2) ; \\
n_{32}^{7}(7,2) \leq 1-y_{2}(6,1)-y_{2}(7,2) .
\end{gathered}
$$

Restrictions 6 (8) are represented by two equations (for two years):

$$
\begin{gathered}
\tilde{x}_{1}(1) \times 150+\tilde{x}_{2}(1) \times 180-y_{1}(3,1) \times 40-y_{1}(4,1) \times 20-y_{2}(5,1) \times 40-y_{2}(6,1) \times 20 \leq 600, \\
\tilde{x}_{1}(2) \times 157+\tilde{x}_{2}(2) \times 189-y_{1}(4,2) \times 20-y_{2}(6,2) \times 20 \leq 900 .
\end{gathered}
$$

Restrictions 4.1 (8) are represented in the problem with 10 equations:

$$
\begin{array}{cc}
y_{1}(3,1) \leq 2 ; & y_{1}(4,2) \leq 2-y_{1}(3,1) ; \\
y_{1}(4,1) \leq 4 ; & y_{1}(5,2) \leq 4-y_{1}(4,1) ; \\
y_{1}(5,1) \leq 5 ; & y_{2}(6,2) \leq 3-y_{2}(5,1) ; \\
y_{2}(5,1) \leq 3 ; & y_{2}(7,2) \leq 1-y_{2}(6,1) ; \\
y_{2}(6,1) \leq 1 ; \\
y_{2}(7,1) \leq 2 .
\end{array}
$$

The conditions for work performance for our problem look like 6 equations given below: $n_{11}^{1}(3,1) \times 38+n_{11}^{2}(3,1) \times 38+n_{11}^{3}(3,1) \times 38+n_{11}^{1}(4,1) \times 30+n_{11}^{2}(4,1) \times 30+n_{11}^{3}(4,1) \times 30+$ $n_{11}^{1}(5,1) \times 20+n_{11}^{2}(5,1) \times 20+n_{11}^{3}(5,1) \times 20+n_{12}^{1}(5,1) \times 11+n_{12}^{2}(5,1) \times 11+n_{12}^{3}(5,1) \times 11+$ $n_{12}^{1}(6,1) \times 6+n_{12}^{2}(6,1) \times 6+n_{12}^{3}(6,1) \times 6+n_{12}^{1}(7,1) \times 4+n_{12}^{2}(7,1) \times 4+n_{12}^{3}(7,1) \times 4+$ $\tilde{x}_{11}^{1}(1) \times 70+\tilde{x}_{11}^{2}(1) \times 70+\tilde{x}_{11}^{3}(1) \times 70+\tilde{x}_{12}^{1}(1) \times 40+\tilde{x}_{12}^{2}(1) \times 40+\tilde{x}_{12}^{3}(1) \times 40 \geq 500$, $n_{22}^{3}(5,1) \times 16+n_{22}^{4}(5,1) \times 16+n_{22}^{5}(5,1) \times 16+n_{22}^{3}(6,1) \times 10+n_{22}^{4}(6,1) \times 10+n_{22}^{5}(6,1) \times 10+$ $n_{22}^{3}(7,1) \times 8+n_{22}^{4}(7,1) \times 8+n_{22}^{5}(7,1) \times 8+\tilde{x}_{22}^{3}(1) \times 55+\tilde{x}_{22}^{4}(1) \times 55+\tilde{x}_{22}^{5}(1) \times 55 \geq 700$, $n_{31}^{6}(3,1) \times 27+n_{31}^{7}(3,1) \times 27+n_{31}^{6}(4,1) \times 20+n_{31}^{7}(4,1) \times 20+n_{31}^{6}(5,1) \times 14+n_{31}^{7}(5,1) \times 14+$ $n_{32}^{6}(5,1) \times 26+n_{32}^{7}(5,1) \times 26+$ $n_{32}^{6}(6,1) \times 19+n_{32}^{7}(6,1) \times 19+n_{32}^{6}(7,1) \times 16+n_{32}^{7}(7,1) \times 16+\tilde{x}_{31}^{6}(1) \times 45+\tilde{x}_{31}^{7}(1) \times 45+$ $\tilde{x}_{32}^{6}(1) \times 60+\tilde{x}_{32}^{7}(1) \times 60 \geq 1000$

$n_{11}^{1}(4,2) \times 30+n_{11}^{2}(4,2) \times 30+n_{11}^{3}(4,2) \times 30+n_{11}^{1}(5,2) \times 20+n_{11}^{2}(5,2) \times 20+n_{11}^{3}(5,2) \times 20+$ $n_{12}^{1}(6,2) \times 6+n_{12}^{2}(6,2) \times 6+n_{12}^{3}(6,2) \times 6+n_{12}^{1}(7,2) \times 4+n_{12}^{2}(7,2) \times 4+n_{12}^{3}(7,2) \times 4+$ $\tilde{x}_{11}^{1}(1) \times 66+\tilde{x}_{11}^{2}(1) \times 66+\tilde{x}_{11}^{3}(1) \times 66+\tilde{x}_{12}^{1}(1) \times 37+\tilde{x}_{12}^{2}(1) \times 37+\tilde{x}_{12}^{3}(1) \times 37+\tilde{x}_{11}^{1}(2) \times 70$ $+\tilde{x}_{11}^{2}(2) \times 70+\tilde{x}_{11}^{3}(2) \times 70+\tilde{x}_{12}^{1}(2) \times 40+\tilde{x}_{12}^{2}(2) \times 40+\tilde{x}_{12}^{3}(2) \times 40 \geq 500$, $n_{22}^{3}(6,2) \times 10+n_{22}^{4}(6,2) \times 10+n_{22}^{5}(6,2) \times 10+n_{22}^{3}(7,2) \times 8+n_{22}^{4}(7,2) \times 8+n_{22}^{5}(7,2) \times 8+$ $\tilde{x}_{22}^{3}(1) \times 51+\tilde{x}_{22}^{4}(1) \times 51+\tilde{x}_{22}^{5}(1) \times 51+\tilde{x}_{22}^{3}(2) \times 55+\tilde{x}_{22}^{4}(2) \times 55+\tilde{x}_{22}^{5}(2) \times 55 \geq 700$, $n_{31}^{6}(4,2) \times 20+n_{31}^{7}(4,2) \times 20+n_{31}^{6}(5,2) \times 14+n_{31}^{7}(5,2) \times 14+n_{32}^{6}(6,2) \times 19+n_{32}^{7}(6,2) \times 19+$ $n_{32}^{6}(7,2) \times 16+n_{32}^{7}(7,2) \times 16+$ 


$$
\begin{gathered}
\tilde{x}_{31}^{6}(1) \times 41+\tilde{x}_{31}^{7}(1) \times 41+\tilde{x}_{32}^{6}(1) \times 56+\tilde{x}_{32}^{7}(1) \times 56+\tilde{x}_{31}^{6}(2) \times 45+\tilde{x}_{31}^{7}(2) \times 45+\tilde{x}_{32}^{6}(2) \times 60 \\
+\tilde{x}_{32}^{7}(2) \times 60 \geq 1000 .
\end{gathered}
$$

In order to simplify the example, let's exclude value $S_{\ni}(m)$ (total operating costs for machine fleet servicing) from the target function. This significantly eases calculations and allows adequately analyzing the problem solving. Thus, the target function will be as follows:

$$
f=\sum_{m}\left[\sum_{j}\left(\tilde{x}_{j}(m) \times \tilde{c}_{j}(m)-\sum_{t \leq \bar{t}_{j}} y_{j}(t, m) \times a_{j}(t, m)\right)\right] \times(1+q)^{1-m} \rightarrow \text { min } .
$$

For the certain example 2 will be as follows:

$$
\begin{aligned}
{\left[\tilde{x}_{1}(1) \times 150+\right.} & \tilde{x}_{2}(1) \times 180-y_{1}(3,1) \times 40-y_{1}(4,1) \times 20-y_{2}(5,1) \times 40-y_{2}(6,1) \\
& \times 20] \times(1+0,2)^{0} \\
& +\left[\tilde{x}_{1}(2) \times 157+\tilde{x}_{2}(2) \times 189-y_{1}(4,2) \times 20-y_{2}(6,2) \times 20\right] \\
& \times(1+0,2)^{-1} \rightarrow \text { min. }
\end{aligned}
$$

After elementary mathematical transformations the target function will be as follows:

$$
\begin{aligned}
\tilde{x}_{1}(1) \times 150+ & \tilde{x}_{2}(1) \times 180-y_{1}(3,1) \times 40-y_{1}(4,1) \times 20-y_{2}(5,1) \times 40-y_{2}(6,1) \\
& \times 20+\tilde{x}_{1}(2) \times 130+\tilde{x}_{2}(2) \times 157-y_{1}(4,2) \times 17-y_{2}(6,2) \times 17 \\
& \rightarrow \min
\end{aligned}
$$

So, we have 102 equations and the target function, in which the controlled variables enter linearly. In other words, we have a linear programming problem for minimum. To solve this problem with the computer, use SIMPLGUS program, the more so, as in a number of publications its advantages in comparison with the other similar programs was noted, major part of which has only demonstration nature.

Optimum solving:

$$
\begin{gathered}
\tilde{x}_{1}(1)=0 ; \\
\tilde{x}_{2}(1)=2.9 ; \\
\tilde{x}_{1}(2)=0 ; \\
\tilde{x}_{2}(2)=2.81 \\
y_{1}(3,1)=y_{1}(4,1)=y_{1}(5,1)=y_{2}(5,1)=y_{2}(6,1)=y_{2}(7,1)=y_{1}(4,2)=y_{1}(5,2)=y_{2}(6,2) \\
=y_{2}(7,2)=0 ; \\
f^{*} \approx 963 \text { m.u. }
\end{gathered}
$$

Optimum values of the basic controlled variables (those ones, which are responsible for equipment purchase and selling) are given above. We are not interested in the variables describing the equipment operation plan in this context.

The algorithm for correction of the optimum solving is given below.

Step 1. Record the controlled variable $\tilde{x}_{2}(1)$ rounding it to three. $\tilde{x}_{2}(1)=3=$ const. Thus, it shall be excluded from (all) restrictions as a variable and shall be included in them (in those ones that contain it) as a constant.

As a result the restrictions included in $\tilde{x}_{2}(1)$ will be as follows:

$$
\begin{gathered}
\tilde{x}_{12}^{1}(1) \leq 3 ; \\
\tilde{x}_{12}^{2}(1) \leq 3 ; \\
\tilde{x}_{12}^{3}(1)+\tilde{x}_{22}^{3}(1) \leq 3 ; \\
\tilde{x}_{22}^{4}(1) \leq 3 ;
\end{gathered}
$$




$$
\begin{gathered}
\tilde{x}_{22}^{5}(1) \leq 3 ; \\
\tilde{x}_{32}^{6}(1) \leq 3 ; \\
\tilde{x}_{32}^{7}(1) \leq 3 ; \\
\tilde{x}_{1}(1) \times 150-y_{1}(3,1) \times 40-y_{1}(4,1) \times 20-y_{2}(5,1) \times 40-y_{2}(6,1) \times 20 \leq 600-540=60 .
\end{gathered}
$$

The target function will be as follows:

$$
\begin{gathered}
\tilde{x}_{1}(1) \times 150-y_{1}(3,1) \times 40-y_{1}(4,1) \times 20-y_{2}(5,1) \times 40-y_{2}(6,1) \times 20+\tilde{x}_{1}(2) \times 130+ \\
\tilde{x}_{2}(2) \times 157-y_{1}(4,2) \times 17-y_{2}(6,2) \times 17+540 \rightarrow \min . \\
f=f_{1}+540 \rightarrow \text { min } \text { or, that is the same as } f_{1} \rightarrow \min ,
\end{gathered}
$$

where $f_{1}=\tilde{x}_{1}(1) \times 150+\tilde{x}_{2}(1) \times 180-y_{1}(3,1) \times 40-y_{1}(4,1) \times 20-y_{2}(5,1) \times 40-y_{2}(6,1) \times 20$ $+\tilde{x}_{1}(2) \times 130-y_{1}(4,2) \times 17-y_{2}(6,2) \times 17$.

The optimum solving after the first correction:

$$
\begin{gathered}
\tilde{x}_{2}(1)=3^{*} ; \quad \tilde{x}_{1}(1)=0 ; \\
\tilde{x}_{2}(2)=2.71 ; \quad \tilde{x}_{1}(2)=0 ; \\
y_{2}(6,2)=0.26 ; \\
y_{2}(7,1)=0.0625 ; \\
y_{1}(5,1)=0.857 ; \\
f^{*} \approx 540+426,5=966,5 \text { m.u. }
\end{gathered}
$$

Step 2 . Round to three $\tilde{x}_{2}(2) . \tilde{x}_{2}(2)=3=$ const. Similarly to the first step of correction, rewrite the restrictions containing the variable $\tilde{x}_{2}(2)$.

$$
\begin{gathered}
\tilde{x}_{12}^{1}(2) \leq 3 ; \\
\tilde{x}_{12}^{2}(2) \leq 3 ; \\
\tilde{x}_{12}^{3}(2)+\tilde{x}_{22}^{3}(2) \leq 3 ; \\
\tilde{x}_{22}^{4}(2) \leq 3 ; \\
\tilde{x}_{22}^{5}(2) \leq 3 ; \\
\tilde{x}_{32}^{6}(2) \leq 3 ; \\
\tilde{x}_{32}^{7}(2) \leq 3 ; \\
\tilde{x}_{1}(2) \times 157-y_{1}(4,2) \times 20-y_{2}(6,2) \times 20 \leq 900-567=333 .
\end{gathered}
$$

The target function will be as follows:

$$
\begin{gathered}
\tilde{x}_{1}(1) \times 150-y_{1}(3,1) \times 40-y_{1}(4,1) \times 20-y_{2}(5,1) \times 40-y_{2}(6,1) \times 20+\tilde{x}_{1}(2) \times 130- \\
y_{1}(4,2) \times 17-y_{2}(6,2) \times 17+\quad 1011 \rightarrow \text { min, or } f_{2} \rightarrow \min ,
\end{gathered}
$$

where $f_{2}=\tilde{x}_{1}(1) \times 150-y_{1}(3,1) \times 40-y_{1}(4,1) \times 20-y_{2}(5,1) \times 40-y_{2}(6,1) \times 20+\tilde{x}_{1}(2) \times 130$ $-y_{1}(4,2) \times 17-y_{2}(6,2) \times 17$.

The optimum solving after the second correction:

$$
\begin{array}{ll}
\tilde{x}_{2}(1)=3^{*} ; & \tilde{x}_{1}(1)=0 ; \\
\tilde{x}_{2}(2)=3^{*} ; & \tilde{x}_{1}(2)=0 ;
\end{array}
$$




$$
\begin{aligned}
& y_{2}(5,1)=0.354 \\
& y_{2}(6,2)=0.393 \\
& y_{1}(3,1)=0.140 \\
& f^{*} \approx 984,5 \text { m.u. }
\end{aligned}
$$

We will perform the step-by-step correction until all controlled variables included into the target function will become integer numbers.

Step 3. Let's assume that $y_{1}(3,1)=0$. Correcting the linear programming problem by the described method and solving it with the computer we obtain the next corrected optimum solving.

$$
\begin{gathered}
\tilde{x}_{2}(1)=3^{*} ; \quad \tilde{x}_{1}(1)=0 ; \\
\tilde{x}_{2}(2)=3^{*} ; \quad \tilde{x}_{1}(2)=0 ; \\
y_{2}(5,1)=0.354 ; \\
y_{2}(6,2)=0.401 ; \\
y_{1}(3,1)=0^{*} ; \\
y_{1}(4,1)=0.190 ; \\
f^{*} \approx 986,2 \text { m.u. }
\end{gathered}
$$

Step 4. $y_{1}(4,1)=0$. The optimum solving after the forth step of correction:

$$
\begin{gathered}
\tilde{x}_{2}(1)=3^{*} ; \quad \tilde{x}_{1}(1)=0 ; \\
\tilde{x}_{2}(2)=3^{*} ; \quad \tilde{x}_{1}(2)=0 ; \\
y_{2}(5,1)=0.354 ; \\
y_{2}(6,2)=0.541 ; \\
y_{1}(3,1)=0^{*} ; \\
y_{1}(4,1)=0^{*} ; \\
f^{*} \approx 987,65 \text { m.u. }
\end{gathered}
$$

Step 5. $y_{2}(5,1)=0$. The optimum solving after the fifth step:

$$
\begin{gathered}
\tilde{x}_{2}(1)=3^{*} ; \quad \tilde{x}_{1}(1)=0 ; \\
\tilde{x}_{2}(2)=3^{*} ; \quad \tilde{x}_{1}(2)=0 ; \\
y_{2}(5,1)=0^{*} ; \\
y_{2}(6,2)=0.418 ; \\
y_{2}(6,1)=0.567 ; \\
y_{1}(3,1)=0^{*} ; \\
y_{1}(4,1)=0^{*} ; \\
f^{*} \approx 992,57 \text { m.u. }
\end{gathered}
$$

Step 6. $y_{2}(6,2)=0$. The optimum solving after the sixth step:

$$
\tilde{x}_{2}(1)=3^{*} ; \quad \tilde{x}_{1}(1)=0 ;
$$




$$
\begin{gathered}
\tilde{x}_{2}(2)=3^{*} ; \quad \tilde{x}_{1}(2)=0 ; \\
y_{2}(5,1)=0^{*} ; \\
y_{2}(6,2)=0^{*} ; \\
y_{2}(6,1)=0.567 ; \\
y_{1}(3,1)=0^{*} ; \\
y_{1}(4,1)=0^{*} ; \\
y_{1}(4,2)=0.397 ; \\
f^{*} \approx 992,92 \text { m.u. }
\end{gathered}
$$

Step 7. $y_{1}(4,2)=0$. The optimum solving after the seventh step:

$$
\begin{gathered}
\tilde{x}_{2}(1)=3^{*} ; \quad \tilde{x}_{1}(1)=0 ; \\
\tilde{x}_{2}(2)=3^{*} ; \quad \tilde{x}_{1}(2)=0 ; \\
y_{2}(5,1)=0^{*} ; \\
y_{2}(6,2)=0^{*} ; \\
y_{2}(6,1)=0.567 ; \\
y_{1}(3,1)=0^{*} ; \\
y_{1}(4,1)=0^{*} ; \\
y_{1}(4,2)=0^{*} ; \\
f^{*} \approx 999,67 \text { m.u. }
\end{gathered}
$$

Step 8. $y_{2}(6,1)=0$. When rounding $y_{2}(6,1)$ to the higher side (to unit) we obtain unsolvability of the first in the problem.

The optimum solving after the eighth step of correction:

$$
\begin{gathered}
\tilde{x}_{2}(1)=3^{*} ; \quad \tilde{x}_{1}(1)=0 ; \\
\tilde{x}_{2}(2)=3^{*} ; \quad \tilde{x}_{1}(2)=0 ; \\
y_{2}(5,1)=0^{*} ; \\
y_{2}(6,2)=0^{*} ; \\
y_{2}(6,1)=0^{*} ; \\
y_{1}(3,1)=0^{*} ; \\
y_{1}(4,1)=0^{*} ; \\
y_{1}(4,2)=0^{*} ; \\
f^{*} \approx 1011 \text { m.u. }
\end{gathered}
$$

\section{Results of Scientific Research}

As a result of the performed research a number of key moments can be distinguished, which confirm "viability" of the economic and mathematical model of reequipment for machinetractor fleet of the agricultural enterprise specified in the earlier publications of the authors. 
Data given in the model example concerning of the technological process and age composition of the equipment in the fleet allowed recording 105 controlled variable of the linear programming problems, as well as restrictions for them. The conditions for work performance by operations are given with six equations, each of which shows data about daily capacity of the machine with the appropriate age and model.

The source record of the problem was transformed into the linear programming problem consisting of 102 equations and target function, in which the controlled variables are included linearly, by simple mathematical transformations.

The procedure of correction was used to the obtained optimum solving of the problem, because as a result non-integral values of basic controlled variables (being responsible for machine purchase and selling) were obtained. The algorithm for correction of the initial optimum solving has already allowed obtaining the optimum problem solving consisting only from integer-values of the controlled variables at the eighth step.

\section{Conclusion}

The model example showed viability economic and mathematical model for reequipment of the machine-tractor fleet at the agricultural enterprise. However, the model testing revealed a number of "weak points" that required correction of the initial solving for the linear programming problem.

As a result of step-by-step correction of the initial solving of the linear programming problem at the eighth step we obtained the optimum plan, in which all basic controlled variables (those ones that are included into the target function) have integer-value nature. According to this plan it is necessary to purchase three second type machines of new modification within the first and second years of the account period accordingly. In the fleet the available equipment shall be discarded only upon achievement of the critical age. Such a variant of reequipment for the fleet will allow performing all required operations within the required scope in time and will cost 1011 monetary units for the enterprise.

\section{References}

1. H. Seyyedhasani, J.S. Dvorak, Biosystems Engineering 169, 1 (2018)

2. C.G. Sorensen, D.D Bochtis, Biosystems Engineering 105, 41 (2010)

3. A. Sopegno, A. Calvo, R. Berruto, P. Busato, D. Bocthis, Computers and Electronics in Agriculture 130, 158 (2016)

4. N.G. Vovchenko, A.A. Alukhanyan, L.Y. Andreeva, G.A. Buryakov, European Research Studies Journal 21, 3 (2018)

5. S. Shepelev, V. Shepelev, Z. Almetova, Procedia Engineering 150, 1258 (2016)

6. D Bochtis, C. G. Sorensen, D. Kateris, Operations Management in Agriculture 117 (2019)

7. D D Bochtis, C. G. Sorensen, P. Busato, Biosystems Engineering 126, 69 (2014)

8. A. Alukhanyan, O. Panfilova, E3S Web of Conferences 175, 13007 (2020)

9. G. Smirnova, R. Sabitov, B. Morozov, S. Sabitov, N. Elizarova, IFAC-PapersOnline 48, 1230 (2015)

10. M. Karunarathna, C. Wilson, Journal of Forest Economics 29, 38 (2017)

11. P. Luukka, M. Collan, Insurance: Mathematics and Economics 65, 22 (2015)

12. Chi-Chuan Lee, Chien-Chiang Lee, Yan-Yu Chiou, Journal of International Financial Markets, Institutions and Money 51, 155 (2017) 
13. G. Reina, A. Milella, R. Galati, Biosystems Engineering 162, 124 (2017)

14. Y. Hu, Y. Liu, Z. Wang, J. Wen, J. Lu, Biosystems Engineering 190, 201 (2020)

15. R.C. Tieppo, T.L. Romanelli, M. Milan, C.G. Sorensen, D. Bochtis, Computers and Electronics in Agriculture 156, 282 (2019)

16. A. Vafador, M. Tolouei-Rad, K. Hayward, K. Abhary, Journal of Manufacturing Systems 39, 53 (2016) 